\title{
Comparison of a practice-based versus theory-based training program for conducting vacuum-assisted deliveries: a randomized-controlled trial
}

\author{
Julian Marschalek ${ }^{1} \cdot$ Lorenz Kuessel $^{1} \cdot$ Maria Stammler-Safar $^{1} \cdot$ Herbert Kiss $^{1} \cdot$ Johannes Ott $^{1} \cdot$ Heinrich Husslein $^{1} \mathbb{C}$
}

Received: 13 March 2021 / Accepted: 21 July 2021 / Published online: 7 August 2021

(c) The Author(s) 2021

\begin{abstract}
Purpose Vacuum-assisted deliveries (VAD) are complex procedures that require training and experience to be performed proficiently. We aimed to evaluate if a more resource intensive practice-based training program for conducting VAD is more efficient compared to a purely theory-based training program, with respect to immediate training effects and persistence of skills 4-8 weeks after the initial training.

Methods In this randomized-controlled study conducted in maternity staff, participants performed a simulated low-cavity non-rotational vacuum delivery before (baseline test) and immediately after the training (first post-training test) as well as 4-8 weeks thereafter (second post-training test). The study's primary endpoint was to compare training effectiveness between the two study groups using a validated objective structured assessment of technical skills (OSATS) rating scale.

Results Sixty-two participants were randomized to either the theory-based group $(n=31)$ or the practice-based group $(n=31)$. Total global and specific OSATS scores, as well as distance of cup application to the flexion point improved significantly from baseline test to the first post-training test in both groups $\left(p_{\text {all }}<0.007\right)$. Skill deterioration after $4-8$ weeks was only found in the theory-based group, whereas skills remained stable in the practice-based group.

Conclusion A practice-based training program for conducting VAD results in comparable immediate improvement of skills compared to a theory-based training program, but the retention of skills $4-8$ weeks after training is superior in a practicebased program. Future studies need to evaluate, whether VAD simulation training improves maternal and neonatal outcome after VAD.
\end{abstract}

Keywords Objective structured assessment of technical skills (OSATS) · Simulation · Training · Vacuum-assisted delivery · Vacuum extraction

\section{Introduction}

Operative vaginal delivery (OVD) with the aim to expedite delivery and thereby reduce maternal and fetal morbidity is an essential skill for obstetricians. The rates of OVD vary worldwide and lie between 3 and 15\% [1,2]. In the last decades, OVD decreased in countries like the United States and Sweden, whereas it increased in countries like Austria or Norway. The choice of instrument (i.e. forceps or vacuum) varies widely around the world, with forceps used in up to

Heinrich Husslein

heinrich.husslein@meduniwien.ac.at

1 Department of Obstetrics and Gynecology, Medical University of Vienna, Spitalgasse 23, 1090 Vienna, Austria
$16 \%$ in primipara in the U.K. compared to less than $0.5 \%$ in Austria and Sweden.

OVDs are complex procedures that require a combination of fine motor skills, understanding of the maternal and fetal anatomy and the mechanics of vaginal birth and therefore need training and experience to be performed proficiently. However, the need for an OVD occurs mostly unscheduled and under stressful circumstances, often caused by maternal or fetal distress. Although the use of a vacuum extractor has been proven to be effective, feasible and safe, its use can provoke significant maternal and fetal morbidity [3-7]. Failed OVD has been directly linked to increased fetal and maternal morbidity and is often attributed to the skill level of the operator $[6,8-10]$. The reported rate of failed vacuumassisted deliveries (VAD) varies throughout the literature and lies between 1 and 34\% [3, 11-14]. 
The traditional approach to teach VAD can be described as "learning-by-doing-model" or a "see one, do oneapproach" under supervision of an expert. However, this approach is no longer appropriate for medical professionals and bears limitations as learning under stressful conditions limits the effectiveness of the educational and learning process $[15,16]$. Acute situations withdraw the timespan to perform tactile trainings or to reconsider and modify the conceptualization. Therefore, theoretical guidelines, training models, and simulators have been evaluated [17-22]. Simulation training has been tested in many fields of medical education and has been shown to improve performance in real clinical situations, especially in procedures that occur rarely or in high-stress environments, making it a potentially ideal modality for VAD training [23, 24]. Nonetheless, due to diminished working hours for trainees and because instructed hands-on-training demands more time and staff resources, theory- and video-based training methods have increasingly aroused interest [17, 21-23].

A recent study tested both models on medical students with no previous exposure to VAD and could demonstrate a significantly higher improvement of VAD skills in the practice-based hands-on-training [17]. However, we do not know whether this is also true in maternity staff with previous exposure to VAD or already skilled in VAD; a key question in efforts aiming to improve the quality of care in obstetric clinical routine.

In this randomized-controlled study conducted in maternity staff, we aimed to evaluate if a practice-based training program for conducting VAD is more efficient compared to a purely theory-based training program, using a validated procedure-specific skill rating scale [25]. Moreover, we intended to measure the persistence of skills 4-8 weeks after the initial training.

\section{Materials and methods}

This randomized-controlled trial was carried out at the Department of Obstetrics and Gynecology, Medical University of Vienna, Austria, between April 2017 and February 2020.

Maternity staff (obstetricians, residents and midwives) were invited to participate in this study.

Oral and written informed consent was obtained from all participants. There were no specific inclusion or exclusion criteria. After enrollment, a unique study identification number was assigned to each participant and participants were randomized into one of the following two groups: (1) a theory-based VAD training program and (2) a theory and practice-based VAD training program. Randomization was carried out by means of serially numbered sealed envelopes, according to a computer-generated randomization plan using a one-to-one randomization. Baseline demographic data, such as age, gender, training status (depending on previously performed VAD), number of previously performed VAD training programs and number of attended vaginal deliveries, were collected.

After randomization, all participants performed a lowcavity non-rotational vacuum delivery in a simulated environment, which served as the baseline test. For all simulation scenarios, the Lucy and Lucy's Mum instrumental delivery birth simulator (Paradigm Medical Systems, Portland, OR) and the Kiwi ${ }^{\circledR}$ Omnicup vacuum extractor (Clinical Innovations, LLC., Putzbrunn, Germany) were used.

After the baseline test, participants allocated to group 1 underwent a 30 -min power-point training session including instructions how to perform a VAD on basis of the technique described by Aldo Vacca [26], followed by a self-guided hands-on training for $15 \mathrm{~min}$. Participants allocated to group 2 underwent the same 30-min power-point training session including the same instructions how to perform a VAD, followed by a one-to-one-instructed hands-on training for $15 \mathrm{~min}$. After the training, every participant performed a second low-cavity non-rotational vacuum delivery on the birth simulator, which served as the first post-training test.

Four to eight weeks after the initial training program, a third low-cavity non-rotational vacuum delivery was performed to determine the persistence of skills ( $=$ second posttraining test).

All simulated vacuum deliveries were recorded using a handheld camera. Participants were filmed in a way, that their identity was not recognizable (i.e., without the participants head). Furthermore, the participants' voices were altered using a software program (Wondershare Filmora 9, Wondershare Software Co., Ltd.).

After having performed all three video-recorded procedures, all participants had the possibility to join a practicebased training program for the first time/or again, depending on the prior randomization. A Consort diagram of the progress through the study is shown in Fig. 1.

The study's primary endpoint was to compare training effectiveness between the two study groups by assessing the videos of the baseline test and the first post-training test of a simulated low-cavity non-rotational vacuum delivery using a validated global and procedure-specific skill rating scale, which was developed on basis of an objective structured assessment of technical skills (OSATS) rating scale [25]. Assessments of the video-recordings were carried out by an obstetrician with 15 years of experience and expert status in conducting vacuum-assisted deliveries. The rater was blinded to the trainee's identity, allocation, experience status as well as the number and order of the video-recordings.

Secondary endpoints included the persistence of training effect and the correct application of the vacuum cup by 
Fig. 1 Consort diagram of the progress through the study

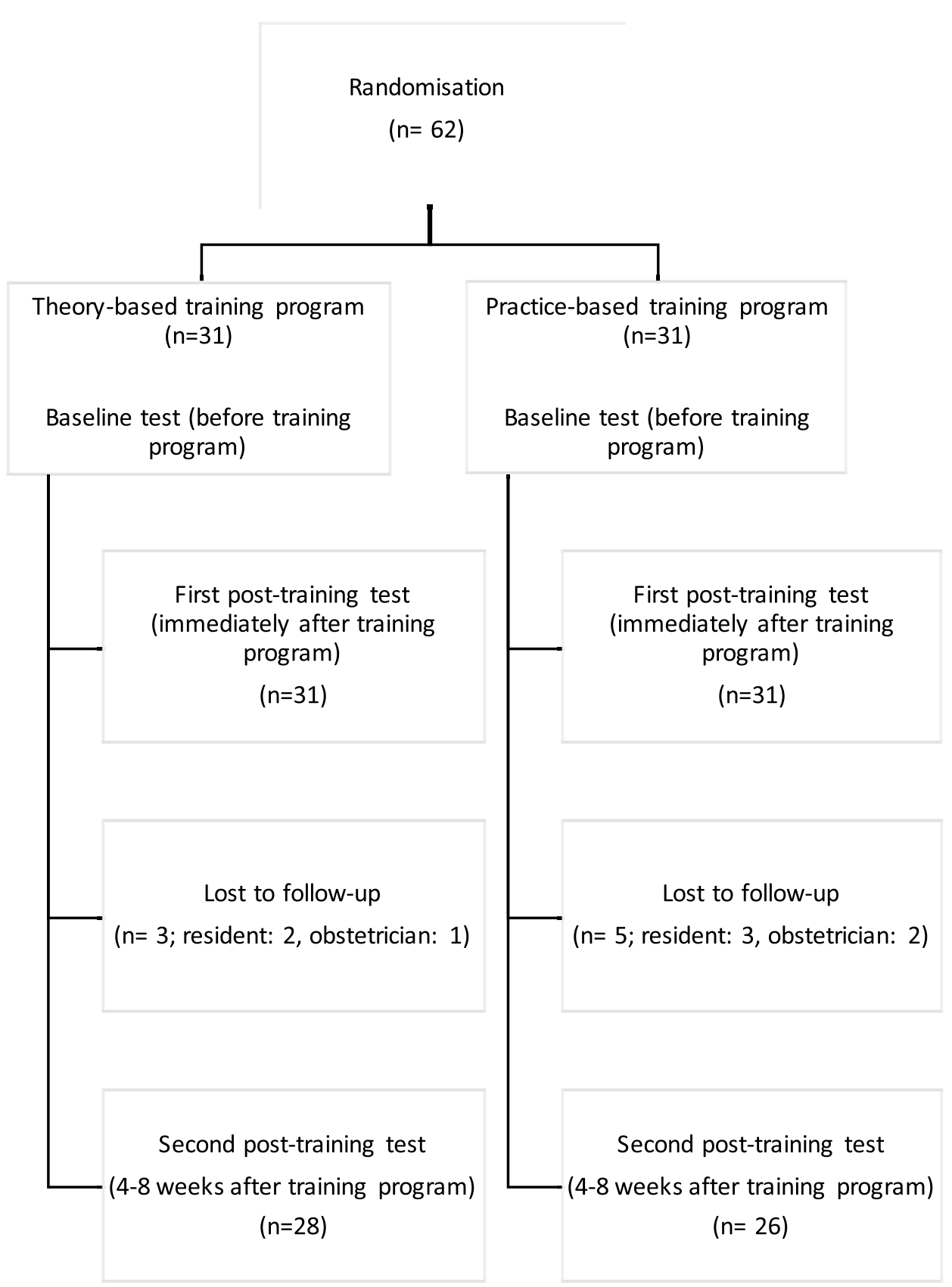

measuring the distance of the cup application to the flexion point at the time of the actual simulations.

With a sample size of $n=30$ subjects per group, we were able to exclude a reasonable difference of total scores (effect size: $d=0.8$ ) with a power of $80 \%$ and a type 1 error rate of $5 \%$ (two-sided), providing an asymptotic relative efficiency for the Wilcoxon-Mann-Whitney test of 0.864 (worst case) as compared to the parametric alternative [27].

The study was conducted in accordance with the Declaration of Helsinki and was registered with Clinical-Trials.gov (NCT03111498). Since the participation was voluntary, the need for an ethical approval for this study was waived by the Ethics Committee of the Medical University of Vienna.

\section{Results}

Sixty-two participants were recruited and randomized to either the theory-based group $(n=31)$ or the practice-based group $(n=31)$. Three participants in the theory-based (and five participants in the practice-based group were lost to follow-up after the first post-training (Fig. 1). There were 
no significant differences between groups concerning demographic characteristics (Table 1).

\section{Baseline test}

There was no difference between the total global and specific OSATS scores between groups. Furthermore, there was no difference regarding the distance of cup application to the flexion point (Supplement Table).

\section{Immediate training effects}

Total global and specific OSATS scores, as well as distance of cup application to the flexion point improved significantly from baseline test to the first post-training test in both groups (Table 2). Improvements were comparable between groups (Table 3).

\section{Long-term training effects}

For evaluation of long-term training effects, baseline tests were compared to the second post-training test after 4-8 weeks. In the practice-based training group, global and specific OSATS scores, as well as distance of cup application to the flexion point were still significantly improved compared to baseline test, whereas in the theory-based practice group, only the specific OSATS scores were still better compared to the baseline test (Table 2).

To demonstrate deterioration of skills over time, we further compared the first (immediate) and second (4-8 weeks after baseline) post-training tests between groups (Table 2). In the practice-based training group, global and specific OSATS scores, as well as distance of cup application to the flexion point remained comparable over time (Table 2). In contrast, in the theory-based practice group, global and specific OSATS scores, as well as distance of cup application to the flexion all deteriorated significantly over time (Table 2). Additionally, we compared the relative difference (delta) of OSATS scores and distance of cup application to the flexion point, revealing a significantly greater deterioration over time in the theory-based group compared to the practice-based group (Table 3).

To determine which procedure-specific skills decreased at first after the training, we compared the procedure-specific ratings scores directly after the training with those 4-8 weeks thereafter in the two groups. In the theorybased group, there was a significant decrease with respect to "vaginal examination for the presenting part, rotation and station" (First post-training test: 4 (IQR 3-5) vs. Second post-training test: 3 (IQR 2-4), $p=0.002$ ), "assessment of the need for oxytocin" (First post-training test: 1 (IQR 1-5) vs. Second post-training test: 1 (IQR 1-1), $p=0.022$ ), "Applies the cup on the flexion point" (First post-training test: 5 (IQR 5-5) vs. Second post-training test: 3.5 (IQR 1.25-5), $p=0.002$ ) and "direction of traction follows the pelvic curve" (First post-training test: 5 (IQR 4-5) vs. Second post-training test: 4 (IQR 2.25-5), $p=0.010$ ). Analysis of the practice-based group revealed only a significant worsening in "protection of the perineum" (First post-training test: 5 (IQR 1-5) vs. Second post-training test: 1 (IQR $1-5), p=0.006)$.
Table 1 Demographic characteristics of the study participants

\begin{tabular}{llll}
\hline & $\begin{array}{l}\text { Theory-based training } \\
(n=31)\end{array}$ & $\begin{array}{l}\text { Practice-based } \\
\text { training } \\
(n=31)\end{array}$ & $p$ Value \\
\hline Age (years) & $27(24-34)$ & $29(25-36)$ & 0.302 \\
Sex & $22(71)$ & $22(71)$ & 1.0 \\
Female & $9(29)$ & $9(29)$ & \\
Male & & & \\
Employees' Status & $20(64.5)$ & $21(67.7)$ & \\
Resident & $9(29)$ & $9(29)$ & $1(3.2)$ \\
Obstetrician & $2(6.5)$ & $19(1-250)$ & 0.463 \\
Midwife & $35(6-200)$ & $12(38.7)$ & 0.797 \\
Attended vaginal births & $14(45.2)$ & $0(0-10)$ & 0.987 \\
Previous exposure to VAD & $0(0-8)$ & $9(29)$ & 0.344 \\
Number of previously performed VAD & & $22(71)$ & \\
Previous Trainings in VAD & $12(38.7)$ & $19(61.3)$ & \\
Yes & & & \\
No & &
\end{tabular}

Categorical data are presented as the frequency and percentage. Continuous variables are expressed as the median and interquartile range (IQR) 


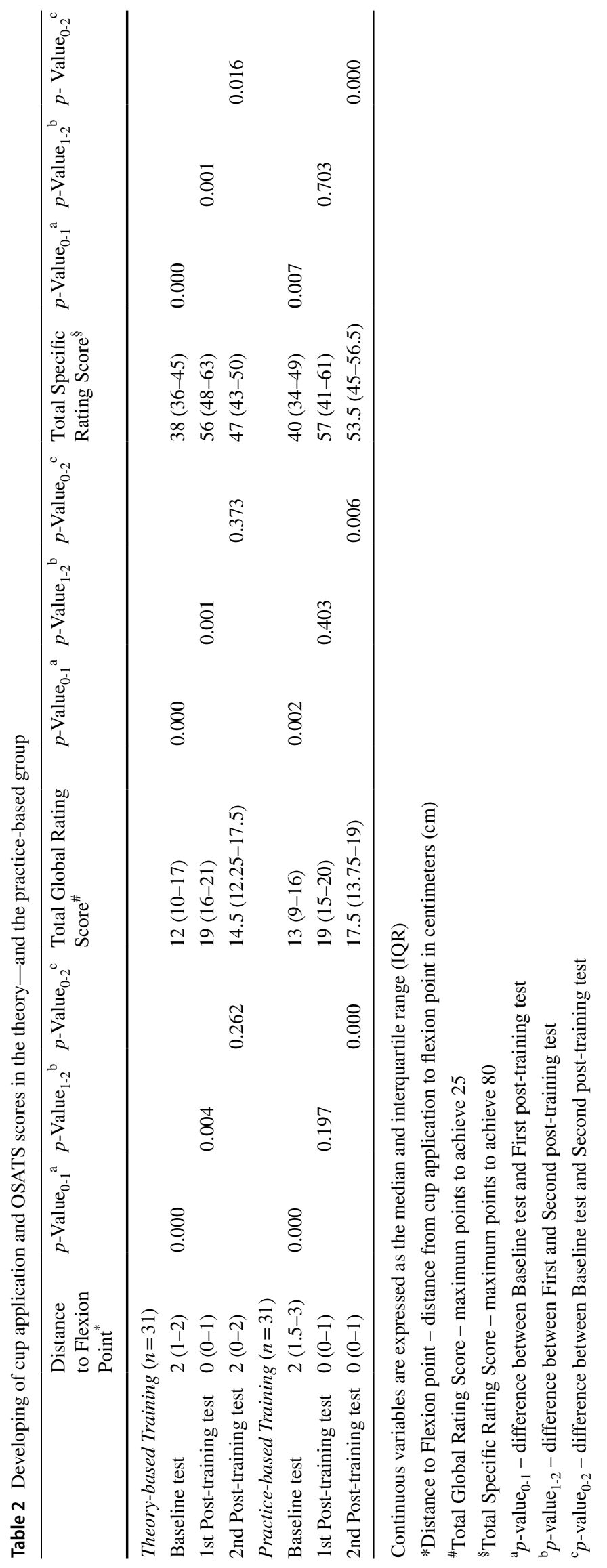


Table 3 Comparing the relative difference (delta, $\Delta$ ) of OSATS scores and cup application between the theory- and the practice-based groups

\begin{tabular}{llll}
\hline & Theory-based training & Practice-based training & $p$ Value \\
\hline $\begin{array}{l}\Delta \text {-Baseline vs. First post-training test } \\
\text { Distance to Flexion Point }\end{array}$ & $-1(-2-0)$ & $-2(-2$ to -1$)$ & 0.137 \\
Total Global Rating Scale & $6(1-9)$ & $5(2-9)$ & 0.972 \\
Total Specific Rating Scale & $10(5-22)$ & $14(7-22)$ & 0.994 \\
$\Delta$-First vs. Second post-training test & & & \\
Distance to Flexion Point & $1(0-2)$ & $0(0-1)$ & 0.028 \\
Total Global Rating Scale & $-4.5(-6.75--1.25)$ & $-1(-5.25-3)$ & 0.039 \\
Total Specific Rating Scale & $-8(-15--0.25)$ & $-2.5(-15-12)$ & 0.082 \\
\hline
\end{tabular}

Continuous variables are expressed as the median and interquartile range (IQR)

\section{Discussion}

The results of our randomized-controlled trial show that a practice-based training program for conducting VAD results in comparable immediate improvement of skills compared to a theory-based training program, but that retention of skills $4-8$ weeks after training is superior in a practice-based program.

Hands-on models have already been shown to significantly enhance the operator's technical skills [23, 24], but so far only few studies evaluating different training programs in VAD exist.

One study including 36 participants reported that a simulation-based training program for VAD resulted in a significant improvement in the accuracy of cup application and theoretical knowledge [28]. Unfortunately, no validated global or procedure-specific skill rating scale was used and no long-term follow-up was performed.

Hilal and colleagues performed a randomized-controlled study comparing a hands-on training versus a video demonstration in medical students with no previous exposure to VAD [17]. They reported a significant improvement in OSATS scores in the hands-on training group immediately after the training and in a second simulation 4-days later. In contrast to our study, they used a self-developed 40-item OSATS scoring system, which was validated in the same study, demonstrating some construct validity. Furthermore, all study participants were medical students with no previous exposure to VAD. Therefore, no baseline test was performed, limiting the information value of the results.

In this study, we have addressed two critical points that, to the best of our knowledge, have not been studied so far. First, whether maternity staff with experience in conducting VAD also show greater improvement from a hands-on training program and second, whether a hands-on training program has a longer-term effect on VAD skills compared to a purely theoretical training program.

Based on our results, both, the theory- and the practicebased training programs lead to significant improvement of global and specific OSATS scores immediately after the training, independently of the trainees' experience status. But only in the practice group, these skills remained 4-8 weeks after the initial training. This long-term effect was also seen when looking at correct cup application: cup application in the second post-training test was significantly more accurate in the practice-based group than in the theorybased group. Of note, to place the cup over the flexion point is a crucial factor for a successful VAD [29, 30]. Correct cup placement is particularly important in mal-positions of the fetal head [26, 31], is reported to reduce "pop-offs" and minimize the duration of the procedure [32, 33]. In contrast, an incorrect cup placement is reported to increase the risk of failed VAD [34] and to contribute to the severity of fetal head traumata, such as cephalohematoma or subgaleal hemorrhage $[35,36]$.

In the theory-based group, OSATS scores of procedurespecific skills decreased after 4-8 weeks in the categories "vaginal examination", "cup application", "direction of traction" as well as "assessment of the need for oxytocin". In the practice-based group, only the category "protection of the perineum" worsened. Specific technical skills, such as correct cup placement over the flexion point and direction of traction, are crucial to reduce the risk of failed VAD and therefore seem to be especially important to retain as long as possible after training [26, 34].

To the best of our knowledge, our study is the first randomized-controlled trial evaluating different VAD training programs conducted in maternity staff reporting on long-term effects assessed by validated OSATS scores. But our study has limitations: first, our study does not provide evidence that the improved skills in a simulation setting translate to an improved maternal and neonatal outcome after VAD. Studies demonstrating transfer of skills from a simulation to a clinical setting are limited [24], but one study reported decreased rates of cervical, severe labial, or high vaginal lacerations as well as less neonatal scalp and facial injury after simulation training in OVD [37]. Second, in the theory-based training program, we used a standardized power-point training session and a single live tutor for the instructions how to perform a VAD. This approach can 
be seen as a limitation due to inter-individual variations or possible differences in the quality of the daily teaching performance. Third, our follow-up period of 4-8 weeks cannot rule out that repetitive trainings might be useful for a long-term persistence of the trainings effect. Of note, a 2012 study evaluating the retention of skills in the management of obstetric complications reported that residents maintained the trainings effect for a year and that repeating the simulation after one year brought additional improvement [38]. This is in accordance with professional societies and guidelines recommending hands-on simulation training of obstetric complications on a yearly basis $[19,39,40]$. If and how a repeated VAD hands-on training proves beneficial one year after the initial training has to be evaluated in future studies.

In conclusion, this study shows that a practice-based training program for conducting VAD results in a prolonged training effect compared to a theory-based training. Especially procedure-specific skills, such as correct cup placement over the flexion point and direction of traction remained stable 4-8 weeks after the hands-on training, whereas they deteriorated after the theory-based training. The immediate training effects were comparable between the practice-based and theory-based training program. Future studies need to evaluate, whether VAD simulation training improves maternal and neonatal outcome after VAD.

Supplementary Information The online version contains supplementary material available at https://doi.org/10.1007/s00404-021-06159-8.

Acknowledgements The authors thank Maria Theresa Trofaiers, M.A. for proof reading the article.

Author contributions JM: protocol development, data collection, manuscript writing/editing; LK: data analysis, manuscript writing/editing; MSS: protocol development, data collection; HK: data collection, manuscript editing; JO: data analysis, manuscript writing; $\mathrm{HH}$ : protocol development, data analysis, manuscript writing/editing.

Funding Open access funding provided by Medical University of Vienna.

Data availability The datasets used and analyzed during the current study are available from the corresponding author on reasonable request.

\section{Declarations}

Conflict of interest JO received remuneration for lecturing from Lenus Pharma GesmbH outside the submitted work. All other authors declare that they have no conflict of interest.

Ethics approval and consent to participate The study was conducted in accordance with the Declaration of Helsinki and was registered in the Current Controlled Trials Register (registration number NCT03111498). Since the participation was voluntary, an approval of the local ethics committee was not required.
Consent for publication All authors have seen and approved the content of the manuscript.

Open Access This article is licensed under a Creative Commons Attribution 4.0 International License, which permits use, sharing, adaptation, distribution and reproduction in any medium or format, as long as you give appropriate credit to the original author(s) and the source, provide a link to the Creative Commons licence, and indicate if changes were made. The images or other third party material in this article are included in the article's Creative Commons licence, unless indicated otherwise in a credit line to the material. If material is not included in the article's Creative Commons licence and your intended use is not permitted by statutory regulation or exceeds the permitted use, you will need to obtain permission directly from the copyright holder. To view a copy of this licence, visit http://creativecommons.org/licenses/by/4.0/.

\section{References}

1. Murphy DJ, Strachan BK, Bahl R, Royal College of Obstetricians and Gynaecologists (2020) Assisted vaginal birth: Greentop Guideline No. 26. BJOG Int J Obstet Gynaecol. https://doi. org/10.1111/1471-0528.16092

2. Ameh CA, Weeks AD (2009) The role of instrumental vaginal delivery in low resource settings. BJOG Int J Obstet Gynaecol 116(Suppl 1):22-25. https://doi.org/10.1111/j.1471-0528.2009. 02331.x

3. Groom KM, Jones BA, Miller N, Paterson-Brown S (2006) A prospective randomised controlled trial of the Kiwi Omnicup versus conventional ventouse cups for vacuum-assisted vaginal delivery. BJOG Int J Obstet Gynaecol 113(2):183-189. https:// doi.org/10.1111/j.1471-0528.2005.00834.x

4. Kuit JA, Eppinga HG, Wallenburg HC, Huikeshoven FJ (1993) A randomized comparison of vacuum extraction delivery with a rigid and a pliable cup. Obstet Gynecol 82(2):280-284

5. Ismail NA, Saharan WS, Zaleha MA, Jaafar R, Muhammad JA, Razi ZR (2008) Kiwi Omnicup versus Malmstrom metal cup in vacuum assisted delivery: a randomized comparative trial. J Obstet Gynaecol Res 34(3):350-353. https://doi.org/10.1111/j. 1447-0756.2007.00701.x

6. Vacca A (2007) Neonatal complications of vacuum-assisted delivery. Obstet Gynecol 110(1):189-189. https://doi.org/10. 1097/01.Aog.0000269870.95850.39

7. Suwannachat B, Lumbiganon P, Laopaiboon M (2012) Rapid versus stepwise negative pressure application for vacuum extraction assisted vaginal delivery. Cochrane Database Syst Rev. https://doi.org/10.1002/14651858.CD006636.pub3

8. Vacca A (2002) Vacuum-assisted delivery. Best Pract Res Clin Obstet Gynaecol 16(1):17-30. https://doi.org/10.1053/beog. 2002.0252

9. Vacca A (2006) Vacuum-assisted delivery: an analysis of traction force and maternal and neonatal outcomes. Aust NZ J Obstet Gynaecol 46(2):124-127. https://doi.org/10.1111/j. 1479-828X.2006.00540.X

10. Towner D, Castro MA, Eby-Wilkens E, Gilbert WM (1999) Effect of mode of delivery in nulliparous women on neonatal intracranial injury. N Engl J Med 341(23):1709-1714. https:// doi.org/10.1056/NEJM199912023412301

11. Bird GC (1982) The use of the vacuum extractor. Clin Obstet Gynaecol 9(3):641-661

12. Johanson RB, Rice C, Doyle M, Arthur J, Anyanwu L, Ibrahim J, Warwick A, Redman CW, O'Brien PM (1993) A randomised prospective study comparing the new vacuum extractor policy with forceps delivery. BJOG Int J Obstet Gynaecol 
100(6):524-530. https://doi.org/10.1111/j.1471-0528.1993. tb15301.x

13. Attilakos G, Sibanda T, Winter C, Johnson N, Draycott T (2005) A randomised controlled trial of a new handheld vacuum extraction device. BJOG Int J Obstet Gynaecol 112(11):1510-1515. https://doi.org/10.1111/j.1471-0528.2005.00729.x

14. Verhoeven CJ, Nuij C, Janssen-Rolf CR, Schuit E, Bais JM, Oei SG, Mol BW (2016) Predictors for failure of vacuum-assisted vaginal delivery: a case-control study. Eur J Obstet Gynecol Reprod Biol 200:29-34. https://doi.org/10.1016/j.ejogrb.2016. 02.008

15. Bahl R, Murphy DJ, Strachan B (2010) Non-technical skills for obstetricians conducting forceps and vacuum deliveries: qualitative analysis by interviews and video recordings. Eur J Obstet Gynecol Reprod Biol 150(2):147-151. https://doi.org/10.1016/j. ejogrb.2010.03.004

16. Roberts MJ, Gale TC, McGrath JS, Wilson MR (2016) Rising to the challenge: acute stress appraisals and selection centre performance in applicants to postgraduate specialty training in anaesthesia. Adv Health Sci Educ Theory Pract 21(2):323-339. https://doi.org/10.1007/s10459-015-9629-6

17. Hilal Z, Kumpernatz AK, Rezniczek GA, Cetin C, Tempfer-Bentz EK, Tempfer CB (2017) A randomized comparison of video demonstration versus hands-on training of medical students for vacuum delivery using Objective Structured Assessment of Technical Skills (OSATS). Medicine 96(11):e6355. https://doi.org/10.1097/ MD.0000000000006355

18. Bahl R, Murphy DJ, Strachan B (2009) Qualitative analysis by interviews and video recordings to establish the components of a skilled low-cavity non-rotational vacuum delivery. BJOG Int J Obstet Gynaecol 116(2):319-326. https://doi.org/10.1111/j.14710528.2008.01967.x

19. ACOG Committee on Obstetric Practice (2006) ACOG Committee Opinion No. 340. Mode of term singleton breech delivery. Obstet Gynecol 108(1):235-237. https://doi.org/10.1097/00006 250-200607000-00058

20. Montanari E, Schwameis R, Louridas M, Gobl C, Kuessel L, Polterauer S, Husslein H (2016) Training on an inexpensive tabletbased device is equally effective as on a standard laparoscopic box trainer: a randomized controlled trial. Medicine 95(39):e4826. https://doi.org/10.1097/MD.0000000000004826

21. Shore EM, Grantcharov TP, Husslein H, Shirreff L, Dedy NJ, McDermott CD, Lefebvre GG (2016) Validating a standardized laparoscopy curriculum for gynecology residents: a randomized controlled trial. Am J Obstet Gynecol 215(2):204 e1-204 e11. https://doi.org/10.1016/j.ajog.2016.04.037

22. Palter VN, Orzech N, Reznick RK, Grantcharov TP (2013) Validation of a structured training and assessment curriculum for technical skill acquisition in minimally invasive surgery: a randomized controlled trial. Ann Surg 257(2):224-230. https://doi.org/10. 1097/SLA.0b013e31827051cd

23. Grantcharov TP, Reznick RK (2008) Teaching procedural skills. BMJ 336(7653):1129-1131. https://doi.org/10.1136/bmj.39517. 686956.47

24. Bligard KH, Lipsey KL, Young OM (2019) Simulation training for operative vaginal delivery among obstetrics and gynecology residents: a systematic review. Obstet Gynecol 134(Suppl 1):16S21S. https://doi.org/10.1097/AOG.0000000000003431

25. Maagaard M, Oestergaard J, Johansen M, Andersen LL, Ringsted C, Ottesen B, Sorensen JL (2012) Vacuum extraction: development and test of a procedure-specific rating scale. Acta Obstet Gynecol Scand 91(12):1453-1459. https://doi.org/10. $1111 / \mathrm{j} .1600-0412.2012 .01526 . x$

26. Vacca A (2009) Handbook of vacuum delivery in obstetric practice. Vacca Research, Albion

27. Hodges JL, Lehmann EL (1955) The efficiency of some nonparametric competitors of the T-test. Ann Math Stat 26(1):155-155

28. Calvert K, Epee M, Karzcub A, Neppe C, Allen M, Hughes W, McGurgan P, King R, Maouris A, Doherty D, Maouris P (2016) Novel simulation workshop improves performance in vacuum delivery. Open J Obstet Gynecol 6:439-444. https://doi.org/10. 4236/ojog.2016.68058

29. Vacca A (2001) Operative vaginal delivery: clinical appraisal of a new vacuum extraction device. Aust NZ J Obstet Gynaecol 41(2):156-160. https://doi.org/10.1111/j.1479-828x.2001.tb012 00.x

30. Yeomans ER (2010) Operative vaginal delivery. Obstet Gynecol 115(3):645-653. https://doi.org/10.1097/AOG.0b013e3181cfbefd

31. Bird GC (1976) The importance of flexion in vacuum extractor delivery. BJOG Int J Obstet Gynaecol 83(3):194-200. https://doi. org/10.1111/j.1471-0528.1976.tb00808.x

32. McQuivey RW (2004) Vacuum-assisted delivery: a review. J Matern Fetal Neonatal Med 16(3):171-180. https://doi.org/10. 1080/1476-7050400001706

33. Plauche WC (1979) Fetal cranial injuries related to delivery with the Malmstrom vacuum extractor. Obstet Gynecol 53(6):750-757

34. Sau A, Sau M, Ahmed H, Brown R (2004) Vacuum extraction: is there any need to improve the current training in the UK? Acta Obstet Gynecol Scand 83(5):466-470. https://doi.org/10.1111/j. 0001-6349.2004.0399.x

35. Doumouchtsis SK, Arulkumaran S (2006) Head injuries after instrumental vaginal deliveries. Curr Opin Obstet Gynecol 18(2):129-134. https://doi.org/10.1097/01.gco.0000192983. 76976.68

36. Baskett TF, Fanning CA, Young DC (2008) A prospective observational study of 1000 vacuum assisted deliveries with the OmniCup device. J Obstet Gynaecol Can 30(7):573-580. https://doi. org/10.1016/S1701-2163(16)32890-0

37. Cheong YC, Abdullahi H, Lashen H, Fairlie FM (2004) Can formal education and training improve the outcome of instrumental delivery? Eur J Obstet Gynecol Reprod Biol 113(2):139-144. https://doi.org/10.1016/S0301-2115(03)00340-3

38. Vadnais MA, Dodge LE, Awtrey CS, Ricciotti HA, Golen TH, Hacker MR (2012) Assessment of long-term knowledge retention following single-day simulation training for uncommon but critical obstetrical events. J Matern Fetal Neonatal Med 25(9):1640 1645. https://doi.org/10.3109/14767058.2011.648971

39. Draycott TJ, Crofts JF, Ash JP, Wilson LV, Yard E, Sibanda T, Whitelaw A (2008) Improving neonatal outcome through practical shoulder dystocia training. Obstet Gynecol 112(1):14-20. https:// doi.org/10.1097/AOG.0b013e31817bbc61

40. Dhingra S, Raffi F (2010) Obstetric trainees' experience in VBD and ECV in the UK. J Obstet Gynaecol 30(1):10-12. https://doi. org/10.3109/01443610903315629

Publisher's Note Springer Nature remains neutral with regard to jurisdictional claims in published maps and institutional affiliations. 